\title{
Inducible and combinatorial gene manipulation in mouse brain
}

\author{
Godwin K. Dogbevia' ${ }^{1,2}$, Ricardo Marticorena-Alvarez ${ }^{3}$, Melanie Bausen ${ }^{1}$, Rolf Sprengel ${ }^{1}$ \\ and Mazahir T. Hasan ${ }^{1,3 *}$
}

${ }^{1}$ Department of Molecular Neurobiology, Max Planck Institute for Medical Research, Heidelberg, Germany, ${ }^{2}$ Institute of Experimental and Clinical Pharmacology and Toxicology, University of Lübeck, Lübeck, Germany, ${ }^{3}$ NeuroCure Cluster of Excellence, Charité-Universitätsmedizin, Berlin, Germany

We have deployed recombinant adeno-associated viruses equipped with tetracyclinecontrolled genetic switches to manipulate gene expression in mouse brain. Here, we show a combinatorial genetic approach for inducible, cell type-specific gene expression and Cre/loxP mediated gene recombination in different brain regions. Our chemicalgenetic approach will help to investigate 'when', 'where', and 'how' gene(s) control neuronal circuit dynamics, and organize, for example, sensory signal processing, learning and memory, and behavior.

Keywords: tetracycline inducible systems, Cre mediated recombination, rAAVs, brain circuits, learning and plasticity, neurological diseases

\section{OPEN ACCESS}

Edited by:

Tycho M. Hoogland,

Netherlands Institute for

Neuroscience, Netherlands

Reviewed by:

Patricia Gaspar,

Institut National de la Santé et de la Recherche Médicale, France

lan Wickersham,

The Salk Institute for Biological

Studies, USA

*Correspondence:

Mazahir T. Hasan,

NeuroCure Cluster of Excellence,

Charité-Universitätsmedizin,

Charitéplatz-1, Virchowweg-6, Berlin,

Germany

mazahir.hasan@charite.de

Received: 09 January 2015

Accepted: 25 March 2015

Published: 21 April 2015

Citation:

Dogbevia GK, Marticorena-Alvarez R,

Bausen M, Sprengel $R$ and Hasan MT (2015) Inducible and combinatorial gene manipulation in mouse brain.

Front. Cell. Neurosci. 9:142.

doi: 10.3389/fncel.2015.00142

\section{Introduction}

Brain is a complex organ, with highly organized genetic and cellular programs that engages neural circuits to generate synaptic specificity (Yogev and Shen, 2014) and plasticity (Citri and Malenka, 2008; Huganir and Nicoll, 2013), controlling a diverse range of biological functions, for example, sensory signal detection, movement, decision-making, and learning and memory (Tonegawa et al., 2003; Kandel et al., 2014).

An attractive hypothesis is that local and distributed neural circuits encode biological functions. To test this hypothesis, it is imperative to 'causally' link the anatomical and functional organization of the neural circuits to biological functions. Manipulating circuit dynamics (Deisseroth, 2014) is paving the way to understand the operating principles of normal and aberrant brain states. Within this framework, understanding the role of gene function(s) in circuit dynamics and biological functions is an important first step in determining the rules and mechanisms that orchestrate changes across different synaptic connections throughout the brain. The application of inducible gene expression systems to manipulate various genes, the building blocks of circuit function(s), in identified brain regions and cell types will help to reveal the molecular and cellular mechanisms that control and regulate biological functions.

The tetracycline (tet) inducible systems (Sprengel and Hasan, 2007) are adequately suitable for investigating various biological processes in the brain. The tet systems have three key components: (1) The transactivator (tTA; Gossen and Bujard, 1992) or the reverse tTA (rtTA; Urlinger et al., 2000), which are artificially designed potent transcription factors that can be expressed constitutively in cells under control of a ubiquitous or a cell type specific promoter. (2) A tet responsive minimal promoter (either unidirectional $P_{\text {tet }}$ or bidirectional $P_{\text {tet }}$ bi; Baron et al., 1995; Sprengel and Hasan, 2007), which become strongly activated upon binding to tTA/rtTA. (3) A chemical inducer, doxycycline (Dox; Bocker et al., 1981), that can rapidly cross the blood-brain-barrier (Bocker et al., 1981), and controls the binding of tTA/rtTA to $\mathrm{P}_{\text {tet }} / \mathrm{P}_{\text {tet }}$ bi. The tTA and $\mathrm{rtTA}$, commonly called Tet-off and Tet-on, respectively, are complementary systems (Sprengel and Hasan, 2007). 
Without Dox, tTA binds to $\mathrm{P}_{\text {tet }} / \mathrm{P}_{\text {tet }}$ bi to activate gene expression. However, when Dox binds to tTA, a conformational change in tTA disables its ability to bind $\mathrm{P}_{\text {tet }} / \mathrm{P}_{\text {tet }} \mathrm{bi}$, inactivating gene expression. The reverse is the case for rtTA; only the Dox-rtTA complex can bind to $\mathrm{P}_{\text {tet }} / \mathrm{P}_{\text {tet }}$ bi to activate gene expression, which becomes inactivated when Dox unbinds rtTA.

In particular, the tTA system has been used in transgenic rodents to repetitively turn 'on' and 'off' gene expression by Dox withdrawal and addition, respectively, (Hasan et al., 2001). The tet systems also allow for gene expression-amplification (Hasan et al., 2004). For inducible gene fragment deletion by Cre/loxP mediated recombination, the tTA system is, however, quite cumbersome. In transgenic tTA mouse models, for Cre/loxP mediated gene manipulation, Cre gene expression in unborn transgenic animals is switched-off by treating pregnant females with Dox. In addition, before investigating specific biological processes, gene expression in newborn pups, is also switched-off by Dox. During both prenatal and pup rearing stages, the Dox reaches the unborn and newly born animals via placenta and milk, respectively. There are two problems with such an approach: (1) Dox availability to the brain via milk is not efficient, which increases the likelihood for undesired Cre/loxP mediated recombination and (2) Dox provided via the placenta in embryos, before blood-brain-barrier is formed, strongly suppresses Cre gene expression, which tend to silence $\mathrm{P}_{\text {tet }} / \mathrm{P}_{\text {tet }}$ bi, disabling gene activation by Dox withdrawal at later stages (Zhu et al., 2007). With excessive accumulation of Dox in tissues during prenatal development, and slow time course of Dox clearance, it can take several months before Cre gene expression can be switched-on by Dox removal. Even after Dox clearance, Cre gene expression in a majority of neurons remains switched-off (Zhu et al., 2007). However, the rtTA system provides a potential solution to this setback. With the rtTA system, Dox activates gene expression via $\mathrm{P}_{\text {tet }} / \mathrm{P}_{\text {tet }}$ bi ( $\mathrm{Zhu}$ et al., 2007). Although the rtTA system works well in various body tissues, its performance is inefficient in the brain of transgenic mammals (Zhu et al., 2007).

In our previous study, we systematically investigated this problem and showed that when tet promoters $\left(\mathrm{P}_{\text {tet }} / \mathrm{P}_{\text {tet }}\right.$ bi) are switched-off during development, they becomes epigenetically silenced in neurons (Zhu et al., 2007). However, introducing a $\mathrm{P}_{\text {tet }}$ bi module in recombinant adeno-associated viruses (rAAVs), which remain largely episomal (Schnepp et al., 2005) in cells, epigenetic silencing of $\mathrm{P}_{\text {tet }}$ bi in neurons can be avoided ( $\mathrm{Zhu}$ et al., 2007). Taking advantage of this discovery, we developed AAVs for reliable and efficient inducible gene expression in brain (Zhu et al., 2007). Our approach is based on two different viruses, which can be delivered in the brain by stereotactic injection (Zhu et al., 2007; Wallace et al., 2008). The first virus delivers in neurons the rtTA (rtTA2-nM2; Zhu et al., 2007) gene under a pan-neuronal human synapsin promoter (Zhu et al., 2007; Cambridge et al., 2009; Hasan et al., 2013). The second virus delivers $\mathrm{P}_{\text {tet }}$ bi to simultaneously express two different genes (tdTomato and iCre; Cambridge et al., 2009; Hasan et al., 2013) in a Dox-controlled, rtTA dependent manner. The AAVs offer other key advantages as well; they allow for precise and combinatorial targeting of brain region(s) at different stage over the course of biological studies, something that is currently not possible with traditional transgenic animal models. The availability of different AAV serotypes and synthetic capsid proteins is expanding the versatility of the AAV-based approach by targeting diverse cell types (Wang et al., 2011; Drouin and Agbandje-McKenna, 2013), with limited or no immune response (Drouin and AgbandjeMcKenna, 2013). Therefore, AAVs have great potentials for investigating basic brain functions and are ideally suited as gene therapy vectors to treat neurological diseases (Bourdenx et al., 2014).

In this study, we deployed AAVs for Dox-controlled, rtTAdependent expression of two genes, Cre (Shimshek et al., 2002) and tdTomato (tdTOM; Shaner et al., 2005). The Cre recombinase allows for Cre/loxP mediated gene manipulation (Capecchi, 2005) of different brain regions and tdTOM marks the cells for live imaging. Our experimental approach will facilitate investigation of gene function(s) in diverse biological processes, including learning and memory (Hasan et al., 2013).

\section{Materials and Methods}

\section{Mice}

C57BL/6N (Charles River, Sulzfeld, Germany) and gene-targeted Gt(ROSA) ${ }^{26 S o r t m 1 S o r / J}$ mice were housed under standard conditions in a $12 \mathrm{~h}$ light/dark cycle in Makrolon cage type-2A with food and water. All animal procedures were performed with permission and in accordance with German governmental regulation on animal experimentation (Regional Council Karlsruhe, Germany: 35-9185.81/G171/10).

\section{Plasmid Constructs}

The plasmids pAAV-hSYN-rtTA2-nM2 (Zhu et al., 2007; Hasan et al., 2013) and pAAV-P tet bi-iCre/tdTOM (Cambridge et al., 2009; Hasan et al., 2013) used here have been described previously. The GenBank Accession Numbers for these plasmids are KP893810 and KP893811, respectively.

\section{Virus Purification}

Serotypes 1 and 2 rAAVs were generated by transfection into HEK293 cells and purified as described previously (Zhu et al., 2007). In brief, each AAV plasmid, pAAV-hSYN-rtTA2nM2 (Zhu et al., 2007; Hasan et al., 2013) and pAAV-P tet $b i-$ iCre/tdTOM (Cambridge et al., 2009; Hasan et al., 2013) was individually co-transfected with $\mathrm{pDp} 1$ (for serotype 1) and $\mathrm{pDp} 2$ (for serotype 2) helper plasmids at a ratio of 2:1:1 per $15-\mathrm{cm}$ plate (25 mg pAAV, $12.5 \mathrm{mg} \mathrm{pDp} 1$ and $12.5 \mathrm{mg} \mathrm{pDp} 2$ ) into HEK293 cells by the DNA/Ca ${ }^{2+} \mathrm{PO}_{4}$ mediated co-precipitation (Chen and Okayama, 1987). We plated HEK cells on twenty 15$\mathrm{cm}$ plates with $25 \mathrm{ml}$ of DMEM medium per plate. On the day of transfection, the cells should be $50 \%$ confluent. Forty-eight hours after transfection ( $50 \%$ efficiency), cells were harvested and collected in $50 \mathrm{ml}$ tubes and pelleted by centrifugation at $2000 \mathrm{rpm}$ for $5 \mathrm{~min}$. The cell pellets were suspended in $45 \mathrm{ml}$ of suspension buffer ( $150 \mathrm{mM} \mathrm{NaCl}, 20 \mathrm{mM}$ Tris. $\mathrm{HCl}, \mathrm{pH} 8.0$ ) and $0.5 \mathrm{ml}$ of $10 \%$ sodium deoxycholate (NaDOC) was added (final concentration of $0.5 \% \mathrm{NaDOC}$ ). To the well-mixed cell 
lysate was added benzonase $(40 \mathrm{U} / \mathrm{ml})$, mixed well, and incubated at $37 \mathrm{C}^{\circ}$ for $60 \mathrm{~min}$. At this stage, cell lysates were frozen at $-70^{\circ} \mathrm{C}$. Before purification, cell lysates were thawed at room temperature and centrifuged at $4000 \mathrm{rpm}$ for $15 \mathrm{~min}$, supernatant collected in a new $50-\mathrm{ml}$ tube and frozen again at $-70^{\circ} \mathrm{C}$ overnight. The next day, supernatant was thawed and centrifuged at $4000 \mathrm{rpm}$ for $15 \mathrm{~min}$. A clear supernatant was passed through a pre-equilibrated 1-ml heparin column (Amersham, Freiburg, Germany) using a pump device. The column was serially washed with $20 \mathrm{ml}$ of $100 \mathrm{mM} \mathrm{NaCl}, 20 \mathrm{mM}$ Tris. $\mathrm{HCl}(\mathrm{pH}), 1 \mathrm{ml}$ of $200 \mathrm{mM} \mathrm{NaCl}, 20 \mathrm{mM}$ Tris. $\mathrm{HCl}$ ( $\mathrm{pH} \mathrm{8}$ ), and $1 \mathrm{ml}$ of $300 \mathrm{mM}$ $\mathrm{NaCl}, 20 \mathrm{mM}$ Tris. $\mathrm{HCl}$ ( $\mathrm{pH}$ 8). The virus was eluted with $1.5 \mathrm{ml}$ of $400 \mathrm{mM} \mathrm{NaCl}, 20 \mathrm{mM}$ Tris. $\mathrm{HCl}$ ( $\mathrm{pH}$ 8), $3 \mathrm{ml}$ of $450 \mathrm{mM}$ $\mathrm{NaCl}, 20 \mathrm{mM}$ Tris. $\mathrm{HCl}$ ( $\mathrm{pH} 8$ ), and $1.5 \mathrm{ml}$ of $500 \mathrm{mM} \mathrm{NaCl}$, $20 \mathrm{mM}$ Tris. $\mathrm{HCl}$ ( $\mathrm{pH} \mathrm{8)}$. The eluted virus was pooled into a 15$\mathrm{ml}$ Amicon Ultra concentrator (Millipore) and filled to the top with 1x PBS. After centrifugation at $2000 \mathrm{rpm}$ for $10 \mathrm{~min}$, the flow through was discarded, and the reservoir was re-filled with $1 \mathrm{x}$ PBS. This procedure was repeated a total of three times. The concentrated viruses $(200 \mu \mathrm{l})$ were sterilized through a $0.2 \mu \mathrm{m}$ small size filter device, aliquoted into eppendorf tubes, and stored at $-80^{\circ} \mathrm{C}$ until use. A small sample of purified virus $(10 \mathrm{ml})$ was analyzed by SDS-PAGE. The gel was stained for $45 \mathrm{~min}$ with Coomassie blue and destained for another $45 \mathrm{~min}$, and washed $5 \mathrm{x}$ with water, until protein bands were clearly visible. With successful virus purification, three bands corresponding to the viral capsid proteins can be seen, with expected molecular weights of $87 \mathrm{kDa}$ (VP1), $73 \mathrm{kDa}$ (VP2), and $62 \mathrm{kDa}$ (VP3).

\section{Responder Virus with Minimal Leakiness}

To reduce leakiness of the $\mathrm{P}_{\text {tet }}$ bi responder virus (rAAV- $\mathrm{P}_{\text {tet }}$ biiCre/tdTOM; Cambridge et al., 2009; Hasan et al., 2013), highly concentrated virus were titered [genomic titer of $1-3 \times 10^{13}$ vector genome (vg) per milliliter]. The responder virus (rAAV$\mathrm{P}_{\text {tet }}$ bi-iCre/tdTOM) was diluted $1: 1,3: 1,10: 1$, and $30: 1$ with respect to the activator virus (rAAV-hSYM-rtTA2-nM2; Zhu et al., 2007; Hasan et al., 2013). About $500 \mathrm{nl}$ of virus cocktail, rAAV $\mathrm{P}_{\text {tet }}$ bi-iCre/tdTOM, and rAAV-hSYN-rtTA2-nM2, was added directly and gently on top of hippocampus organotypic brain slices. Medium was changed after 5 days and, subsequently, every 3 days. Two weeks after virus infection, two slices were incubated with $1 \mu \mathrm{g} / \mathrm{ml}$ Dox in the medium for $48 \mathrm{~h}$ and two slices were kept without Dox (control). Forty-eight hours after Dox addition, tissues were washed in warm 1x PBS and fixed in $4 \%$ PFA. Fixed tissues were imaged for tdTOM expression. The diluted virus cocktail that showed undetectable tdTOM expression (without Dox) and strong tdTOM expression with Dox was chosen for in vivo application.

\section{Stereotactic Virus Injection in Mouse Brain}

C57BL/6N and Gt(ROSA) 26 Sortm 1 Sor/J mice were deeply anesthetized with ketamine $(100 \mathrm{mg} / \mathrm{kg})$ and xylazine $(5 \mathrm{mg} / \mathrm{kg})$, and were secured in a Kopf stereotaxic setup (Kopf Instruments, Tujunga, CA, USA). The foreskin on the skull was cut open to expose the skull. A small hole $(50-100 \mu \mathrm{m})$ was made through the skull using a dental drill. A glass pipette delivered approximately $200 \mathrm{nl}$ of virus cocktail by injection into different brain regions; cortex and hippocampus. The coordinates used for the injections are with reference to the bregma: cortex $(-1.70 \mathrm{~mm}$ bregma, $1.5 \mathrm{~mm}$ lateral, $500 \mu \mathrm{m}$ deep) and hippocampus $(-1.70 \mathrm{~mm}$ bregma, $1.5 \mathrm{~mm}$ lateral, $1.5 \mathrm{~mm}$ deep). After virus injection, the skin was sutured and the wound was disinfected. Virus injected mice were kept on a heating blanket at $37^{\circ} \mathrm{C}$ until they woke up, and were fed wet food during recovery.

\section{Dox Treatment In Vivo}

For intraperitoneal injection, stock Dox solution $(5 \mathrm{mg} / \mathrm{ml}$ in $0.9 \% \mathrm{NaCl}$ ) was prepared. Effective dose is $50 \mu \mathrm{g}$ of Dox per gram body weight (10 $\mu$ l of Dox per $1 \mathrm{~g}$ of animal weight).

\section{Fixed Brain Slices}

Mice were anesthetized by isoflurane inhalation followed by intracardial perfusion with PBS and 4\% paraformaldehyde (PFA). Brains were removed and post-fixed in $4 \%$ PFA for $2 \mathrm{~h}$ at $4^{\circ} \mathrm{C}$, kept in $1 \mathrm{x}$ PBS at $4^{\circ} \mathrm{C}$ overnight, washed $3 \mathrm{x}$ with $\mathrm{PBS}$ and embedded in $2.5 \%$ agarose (in $1 \mathrm{x}$ PBS). Typically, we prepared vibratome (VT1000S, Leica, Wetzlar, Germany) brain slices, which were $60-100 \mu \mathrm{m}$ in thickness. Coronal brain slices were stored in $1 \mathrm{x}$ PBS at $4^{\circ} \mathrm{C}$.

\section{Immunohistochemistry}

Brain slices were incubated in $4 \%$ normal goat serum (supplemented with $1 \%$ BSA, $0.3 \%$ TritonX-100) for $15 \mathrm{~min}$ and then incubated overnight at room temperature with primary Cre-antibody (mouse monoclonal anti-Cre; 1:1000, Covance, Germany) diluted in PBS/ 1\% BSA/ 1\% normal goat serum/ $0.3 \%$ TritonX-100. The next day, brain slices were washed $2 \mathrm{x}$ in 1x PBS/ 0.3\% BSA/ 0.1\% TritonX-100 (D2), followed by incubation with anti-mouse FITC secondary antibody (1:200, Jackson Immuno Research) for $1 \mathrm{~h}$ at room temperature. Brain slices were washed in 1x D2 buffer followed by a single wash in 1x PBS and mounted with Aqua Poly/Mount on glass slides with coverslips.

\section{$\beta$-Galactosidase Assay}

rAAV-hSYN-rtTA2-nM2 (Zhu et al., 2007; Hasan et al., 2013) and rAAV-P $\mathrm{P}_{\text {tet }}$ bi-iCre/tdTOM (Cambridge et al., 2009; Hasan et al., 2013) were co-injected into the hippocampus and cortex of Gt(ROSA) 26 Sortm 1 Sor/J mice. Two weeks after virus injection, mice were either not treated or treated with Dox by a single intraperitoneal injection. After $48 \mathrm{~h}$, animals were sacrificed, brains were fixed and sliced as described above. To visualize $\beta$ galactosidase activity, fixed brain slices were incubated in X-gal solution $\left(5 \mathrm{mM} \mathrm{K}_{4} \mathrm{Fe}(\mathrm{CN})_{6}, 5 \mathrm{mM} \mathrm{K}_{3} \mathrm{Fe}(\mathrm{CN})_{6}, 2 \mathrm{mM} \mathrm{MgCl}_{2}\right.$, $2 \mathrm{mg} / \mathrm{ml} \mathrm{X}$-Gal in dimethylformamide/PBS) at room temperature for 30-60 min. Sections were washed 3x in PBS, $1 \mathrm{x}$ in $10 \mathrm{mM}$ Tris- $\mathrm{HCl} \mathrm{pH} 7.5$ and, subsequently, mounted on glass slides with Aqua Poly/Mount (Polysciences, Inc., Warrington, PA, USA) and protected with cover slips and later imaged.

\section{Imaging}

Light and fluorescence imaging were performed with Zeiss Axioplan-2 (Carl Zeiss, Jena, Germany) with the camera system AxioCam HRC with magnifications ranging from $2.5 \times$ to $40 \times$ 
dry or $63 \times$ oil immersion objectives (software: Axiovision 4.8.1) and a compact light source (Leistungselectronic Jena, Germany) with $488 \mathrm{~nm}$ and $568 \mathrm{~nm}$ filters. Confocal images were acquired with Zeiss LSM PASCAL confocal laser-scanning microscope equipped with an Argon laser (457, 476, 488, and $514 \mathrm{~nm}$ ) and a Helium Neon laser $(543 \mathrm{~nm})$ with objectives $5 \times-40 \times$ dry and $63 \times$ oil-immersion objective. Images were analyzed with ImageJ and LSM image browser.

\section{Results}

\section{Gene Activation with Tet Promoters}

To achieve Tet-inducible gene expression and gene deletion in mouse brain, we used two rAAVs, rAAV-hSYN-rtTA2-nM2 (Zhu et al., 2007; Hasan et al., 2013) and rAAV-P tet bi-iCre/tdTOM (Zhu et al., 2007; Cambridge et al., 2009; Hasan et al., 2013), and loxP-STOP-loxP-lacZ reporter (Gt(ROSA) 26 Sortm 1 Sor $/ J$; Soriano, 1999) transgenic mice (Figures 1A,B). The first virus (rAAVhSYN-rtTA2-nM2) is equipped with the human synapsin promoter to drive rtTA (rtTA2-nM2) expression. The second virus (rAAV-P tet bi-iCre/tdTOM) has a bidirectional tet promoter $\left(\mathrm{P}_{\text {tet }}\right.$ bi) to express two responder genes, iCre (Shimshek et al., 2002) and tdTomato (Shaner et al., 2005; tdTOM; Figure 1A). These two viruses were injected in the cortex of four wild-type mice. Two weeks later, two mice were treated with Dox by a single intraperitoneal injection and the other two mice were not treated with Dox. We found that there was robust expression of tdTOM and Cre recombinase 2 days after Dox treatment. In the cortex, expression was largely restricted to neurons of layer $2 / 3$ and 5 , which was evenly distributed in soma and dendrites, but there was little or no expression in cortical layers 4 and 6 neurons. No tdTOM and Cre expression was detectable in mice without Dox. The two transgenes (tdTOM and iCre) under a $\mathrm{P}_{\text {tet }}$ bi also showed faithful co-expression (Figure 2A). Interestingly, when one gene (GFP variant) was placed under control of a $\mathrm{P}_{\text {tet }}$ bi (rAAV- $\mathrm{P}_{\text {tet }}$ biGFPvariant, called here rAAV-uni- $\mathrm{P}_{\text {tet }}-\mathrm{GFPvariant)}$, strong gene expression was observed, even without Dox (Figure 2B), and was quite comparable to tdTOM expression under a constitutive human synapsin promoter (Figure 2B). We made this observation with three different GFP-linked genes with a similar rAAV-uni-P tet-GFPvariants (data not shown). These results suggest that the placement of two genes flanking a $\mathrm{P}_{\text {tet }}$ bi module allow for Dox-controlled, rtTA-dependent regulated gene expression, but not when only one gene is placed under a structurally intact $\mathrm{P}_{\text {tet }}$ bi.

\section{Time Course of Gene Activation}

To investigate the time course of Dox-controlled, rtTAdependent gene activation in vivo, the two viruses (Zhu et al., 2007; Cambridge et al., 2009; Hasan et al., 2013; rAAV-hSYNrtTA2-nM2 and rAAV-P tet bi-iCre/tdTOM) were co-injected into the cortex of 10 wild-type mice. Two weeks later, mice were divided into five groups (two mice per group). The first group was without Dox (control) and the other four groups were treated with Dox with a single intraperitoneal injection, and tdTOM expression was analyzed after $6,12,24$, and $48 \mathrm{~h}$ in fixed brain slices. While tdTOM expression was not detectable in mice without Dox (Figure 3, left panel), Dox-treated mice showed strong expression as early as $6 \mathrm{~h}$, and maximum expression was reached after $24 \mathrm{~h}$ (Figure 3).

\section{Inducible Gene Expression and Cre/LoxP Mediated Gene Recombination}

To achieve neuron- and brain region-specific inducible gene expression and Cre/loxP mediated gene recombination, viruses (Zhu et al., 2007; Cambridge et al., 2009; Hasan et al., 2013; rAAV-hSYN-rtTA2-nM2 and rAAV-P tet bi-iCre/tdTOM) were co-injected in two different brain regions in three different combinations; cortex alone, hippocampus alone, and both cortex and hippocampus (two mice per group). Two weeks after virus injection, mice were treated with Dox by a single intraperitoneal injection. Two mice served as controls (without Dox). Two days after Dox injection, fixed brain slices showed strong tdTOM
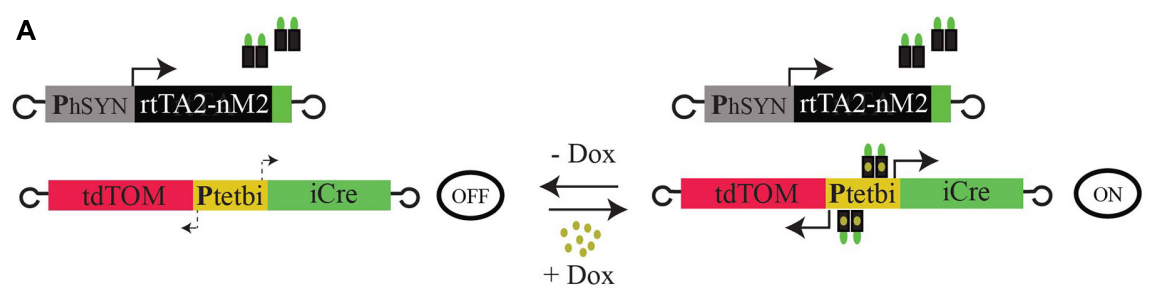

B
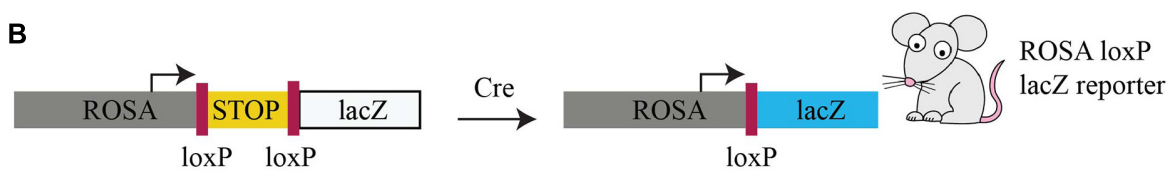

FIGURE 1 | Operating principles. (A) Schematics of rAAV-rtTA system; a virus with a human synapsin promoter for constitutive, pan-neuronal specific rtTA expression and another virus with a bidirectional tetracycline (tet) promoter ( $P_{\text {tet }}$ bi) driving two different genes (tdTOM and iCre) in opposite directions. Binding of doxycycline (Dox) to rtTA enables it to bind to $\mathrm{P}_{\text {tet }} \mathrm{bi}$, and gene expression is bidirectionally switched-ON. In the absence of Dox, rtTA is unable to bind $P_{\text {tet }}$ bi and gene expression is switched-OFF. (B) Schematic of the lacZ Cre-dependent reporter in Gt(ROSA) ${ }^{26 S o r t m 1 S o r / J}$ mice. The ROSA promoter drives the expression of the lacZ gene, but this expression is blocked by transcriptional terminator sequences (STOP), which are flanked by loxP sites. The terminator STOP fragment is removed by Cre/loxP mediated gene deletion to activate the expression of the lacZ gene. 


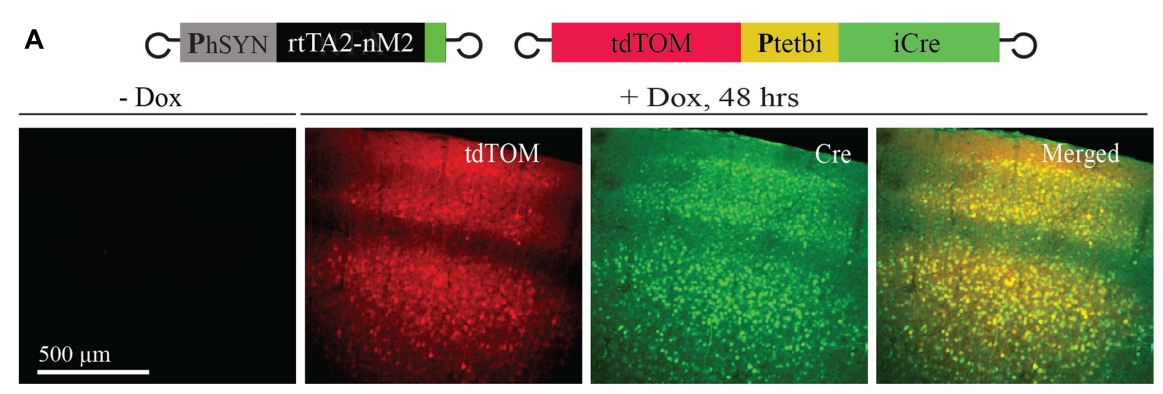

B

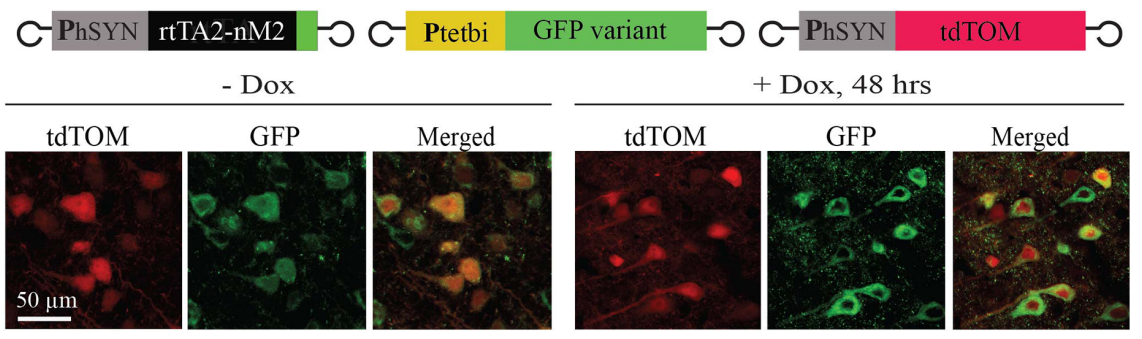

FIGURE $2 \mid \mathbf{P}_{\text {tet }}$ bi assisted inducible gene expression. (A) After Dox treatment, the $\mathrm{P}_{\text {tet }} \mathrm{bi}-\mathrm{iCre} / \mathrm{tdTOM}$ vector enabled robust tdTOM and iCre co-expression in an rtTA dependent manner (right), but not without Dox (left). Both tdTOM and iCre expression was colocalized in cortical neurons (right; merged image). (B) Expression under the control of Ptetbi-GFP-variant vector alone was similar, both with and without Dox, and it compared well with tdTOM expression under the human synapsin promoter.

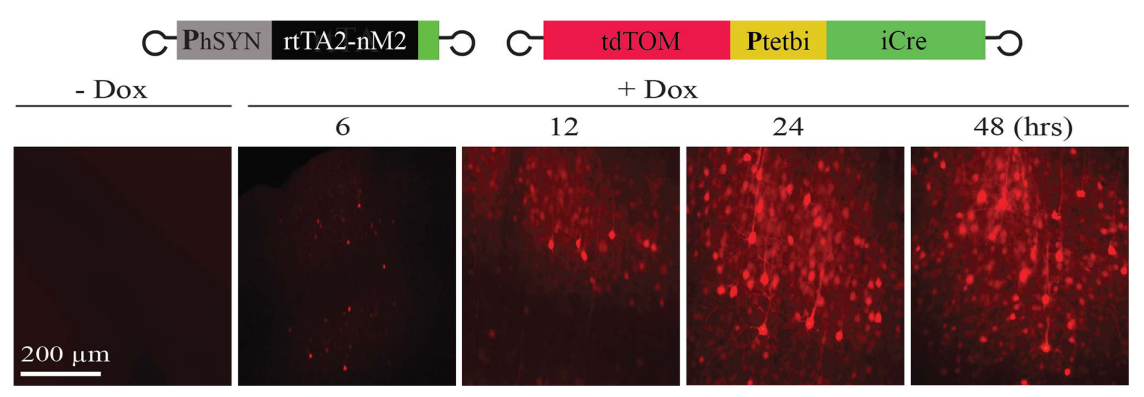

FIGURE 3 | Time course of gene activation. After Dox treatment, gene activation was detected in vivo as early as $6 \mathrm{~h}$, which reached maximum levels in the cortex after $24 \mathrm{~h}$.

expression (Figure 4A). In the hippocampus, expression was widespread in the dentate gyrus granule cells, and the CA1/CA3 pyramidal neurons. No tdTOM expression was detected in mice without Dox (Figure 4A). To test for Cre/loxP gene recombination in specific brain regions in the Gt(ROSA) 26 Sortm 1 Sor $/ J$ mice, viruses (rAAV-hSYN-rtTA2-nM2 and rAAV- $\mathrm{P}_{\text {tet }}$ bi-iCre/tdTOM) were co-injected again in three different combinations; cortex alone, hippocampus alone, and both cortex and hippocampus (two mice per group). Two weeks after virus injection, mice were injected with a single dose of Dox and two mice were used as control (without Dox). Forty-eight hours later, fixed brain slices showed strong $\beta$-galactosidase activity by $\mathrm{X}$-gal staining (Figure 4B), indicating that Cre/lox $\mathrm{P}$ mediated gene recombination occurred efficiently in Dox-treated mice. It should be noted, however, that if the viruses do not reach the entire brain structure, as in the case of hippocampus alone example, a small region would remain untargeted (see CA2 region, Figure 4B, lower right panel). Control mice, without Dox, showed recombination in only a few cells (Figure 4B, left panel).

\section{Discussion}

Here, we report a versatile genetic approach that takes advantage of two rAAVs for inducible, brain region, and cell type specific gene manipulation. Our proof-of-principle approach is currently based on the tet inducible gene expression system, namely the rtTA system, for Dox-induced Cre recombinase expression to induce Cre/loxP mediated gene recombination. The first virus is equipped with a cell type specific promoter to express rtTA (rtTA2-nM2; Zhu et al., 2007). The second virus harbors a bidirectional tet promoter $\left(\mathrm{P}_{\text {tet }} \mathrm{bi}\right)$ to co-express two different genes 

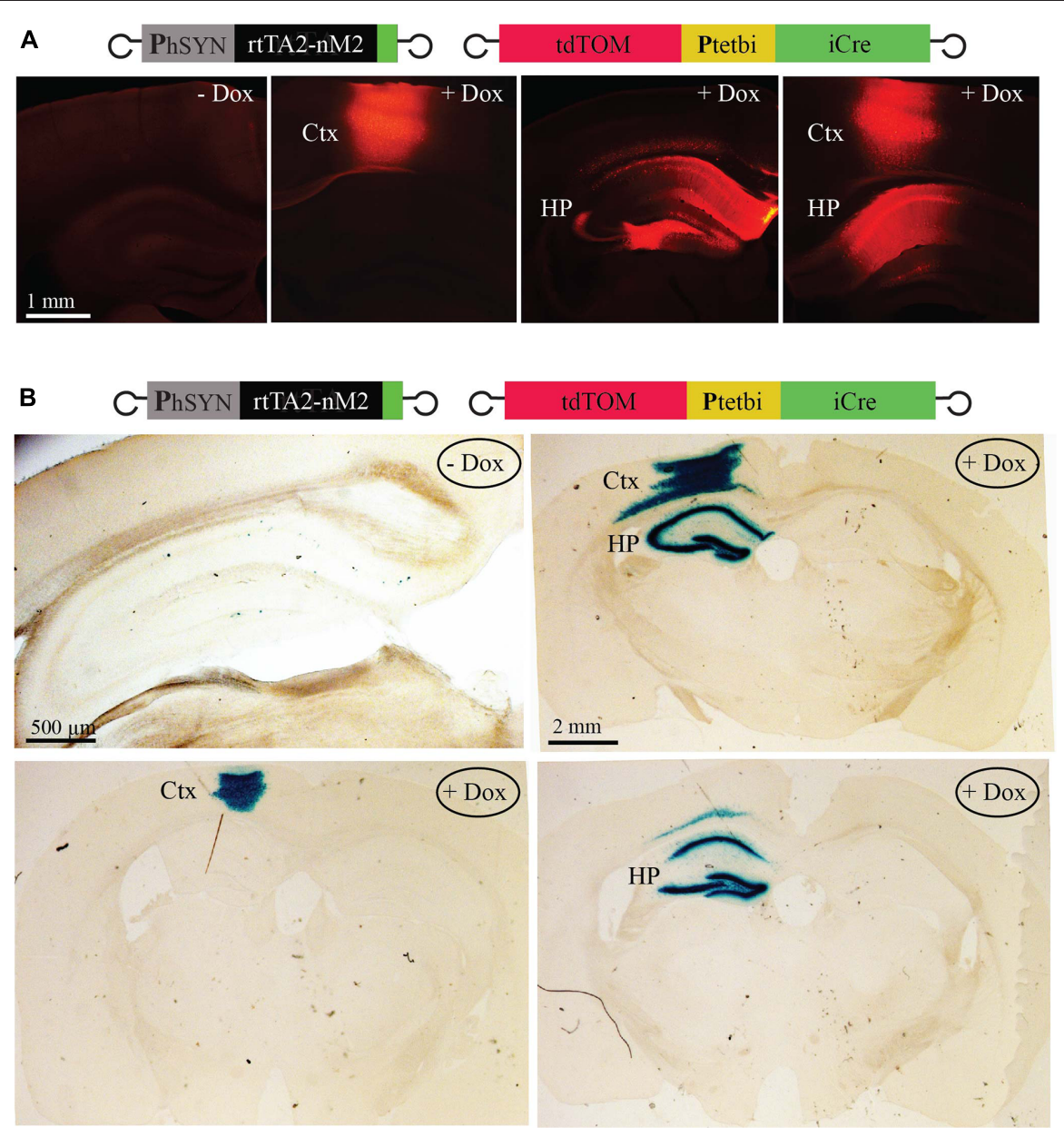

FIGURE 4 | Inducible, Cre/loxP mediated gene recombination.

(A) With precisely targeted virus injection, induced tdTOM expression in cortex alone, hippocampus alone and both cortex and hippocampus. (B) Cre/loxP mediated recombination to activate the lacZ gene in different singly targeted brain region and in combination of two regions. Expression of $\beta$-galactosidase was assayed by $\mathrm{X}$-gal staining. Very little recombination was detectable without Dox (left panel). Ctx, cortex; HP, hippocampus. in opposite orientations (Baron et al., 1995). The use of a fluorescent protein in $\mathrm{P}_{\text {tet }}$ bi, for example, tdTOM (Shaner et al., 2005), helps to monitor the expression of a second gene in the targeted brain region(s). Inducible activation of gene expression via a $\mathrm{P}_{\text {tet }}$ bi requires an rtTA and an inducer, Dox, which can be delivered to the animals by an intraperitoneal injection (Zhu et al., 2007; applied here) and/or in the drinking water (not applied here). We found that Dox-induced, rtTA-dependent $\mathrm{P}_{\text {tet }}$ bi mediated gene expression in targeted neurons can be detected within, at least, a few hours. Our AAVs are of a hybrid serotype (1/2), which appear to largely target layer $2 / 3$ and layer 5 neurons, but not layer 4 and layer 6 neurons. We have not observed retrograde labeling with the AAV1/2.

We demonstrate the applicability of virus approach for inducible gene expression and Cre/loxP mediated gene recombination in the brain of lac $Z$ transgenic reporter mice [Gt(ROSA) ${ }^{26 S o r t m 1 S o r / J}$; Soriano, 1999]. It is well established that the lac $Z$ transgenic reporter mice is a reliable model for natural floxed genes. Our approach can also be extended to overexpress wild-type and mutant genes (Eschbach and Danzer, 2014) and interference RNA (Murphy et al., 2013; Ramachandran et al., 2013). With stereotactic virus injection, we can achieve longterm gene expression in either a single or multiple brain regions, enabling systematic investigation of how different brain regions participate in various biological processes, for example, learning and memory.

We found that rAAVs equipped with minimal tet promoters $\left(\mathrm{P}_{\text {tet }} / \mathrm{P}_{\text {tet }}\right.$ bi $)$ are not without problems. First, $\mathrm{P}_{\text {tet }} / \mathrm{P}_{\text {tet }}$ bi have very low levels of intrinsic transcriptional activity, which is one source of leakiness. In most cases, this is not a major problem. However, with a high virus titer, rAAV- $\mathrm{P}_{\text {tet }}$ bi-iCre/tdTOM (Zhu et al., 2007; Cambridge et al., 2009; Hasan et al., 2013) alone (without rtTA and without Dox), for example, can produce enough Cre recombinase protein in a small number of neurons, particularly, at the virus injection brain site(s) to allow for Cre/loxP mediated gene recombination (data not shown). It is therefore important to serially dilute $\mathrm{P}_{\text {tet }}$ bi viruses with a constant amount of an rtTA (or tTA) virus and only apply an optimal virus 
cocktail $\left(\mathrm{P}_{\text {tet }} \mathrm{bi}+\mathrm{rtTA}\right)$ for efficient and reliable Dox-induced, rtTA-depednent Cre/loxP mediated gene recombination.

The other issue is that the two flanking inverted terminal repeats (ITRs; Bohenzky et al., 1988) in rAAVs appear to have a cryptic enhancer activity. We found that when one gene is placed under a $\mathrm{P}_{\text {tet }}$ bi (equivalent to $\mathrm{P}_{\text {tet }}$ ), it becomes highly active. We reasoned that this increase in $\mathrm{P}_{\text {tet }}$ bi activity was influenced by a nearby ITR. It is known that minimal promoters including $\mathrm{P}_{\text {tet }} / \mathrm{P}_{\text {tet }}$ bi can trap enhancers (Stanford et al., 2001). We thus speculate that an ITR can act in cis to increase the basal activity of the minimal tet promoter. This phenomenon might also explain why gene expression modules flanked by ITR sequences in transgenic zebrafish enabled stable and uniform gene expression (Hsiao et al., 2001), throughout generations, but not without ITRs (Hsiao et al., 2001). In our approach, two different genes

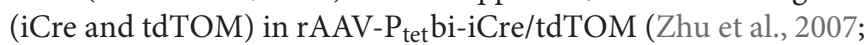
Cambridge et al., 2009; Hasan et al., 2013) appear to shield $\mathrm{P}_{\text {tet }}$ bi from ITR enhancer-like activity; adding DNA sequences of more than $700 \mathrm{bp}$ in between a $\mathrm{P}_{\text {tet }}$ bi appears to minimize the influence of the two ITRs onto $\mathrm{P}_{\text {tet }}$ bi. Clearly, the proposed role of an ITR as an enhancer should be investigated more systematically. It still remains an open question, however, if different gene fragments might insulate $\mathrm{P}_{\text {tet }}$ from an adjacent ITR to a different extent.

Ours is not the first example of a two AAV approach for inducible gene expression. A previous study elegantly used tet transsilencer (tTS) and rtTA on one virus and a $\mathrm{P}_{\text {tet }}$ (unidirectional) on another virus to express GFP in a Dox-controlled, rtTA-dependent manner (McGee Sanftner et al., 2001). In that system, in the absence of Dox, the tTS prevents leaky expression by $\mathrm{P}_{\text {tet }}$, possibly by blocking the ITR enhancer-like activity. With Dox treatment, tTS come off, and rtTA binds to $\mathrm{P}_{\text {tet }}$ to activate gene expression. The important question is how much leakiness was actually prevented by tTS, so that Cre/loxP mediated recombination would only occurs upon Dox treatment.

The major advantage of our two-virus approach is that either single or multiple brain regions can be targeted for inducible and cell type specific gene manipulation by Cre/loxP mediated gene recombination. The major drawback is that a single virus injection can only target a small brain region, but it has the capability to target larger areas by multiple virus injections (Hasan et al.,

\section{References}

Baron, U., Freundlieb, S., Gossen, M., and Bujard, H. (1995). Co-regulation of two gene activities by tetracycline via a bidirectional promoter. Nucleic Acids Res. 23, 3605-3606. doi: 10.1093/nar/23.17.3605

Bocker, R., Estler, C. J., Maywald, M., and Weber, D. (1981). Comparison of distribution of doxycycline in mice after oral and intravenous application measured by a high-performance liquid chromatographic method. Arzneimittelforschung 31, 2116-2117.

Bohenzky, R. A., LeFebvre, R. B., and Berns, K. I. (1988). Sequence and symmetry requirements within the internal palindromic sequences of the adenoassociated virus terminal repeat. Virology 166, 316-327. doi: 10.1016/00426822(88)90502-8

Bourdenx, M., Dutheil, N., Bezard, E., and Dehay, B. (2014). Systemic gene delivery to the central nervous system using Adenoassociated virus. Front. Mol. Neurosci. 7:50. doi: 10.3389/fnmol.2014. 00050
2013). The inducible genetic switches in our viruses provide an added advantage over a single virus approach for constitutive Cre recombinase expression; with our approach, Cre/loxP mediated gene recombination can be activated by Dox treatment after a particular biological process, such as memory formation, without causing stress to animals by a surgical intervention for virus injection, thus avoiding potential stress-related effects. Given that targeting selective brain region(s) for gene expression/manipulation is a major hurdle with the traditional transgenic, our virus-based approach can be of a great value for neuroscience research and gene therapy.

\section{Conclusion}

In summary, we show here a two-virus approach for inducible gene expression and Cre/loxP mediated gene recombination in the mouse nervous system. Our approach makes it possible to target a single or multiple brain regions, and thus provides the neuroscience community with an important genetic tool to investigate how gene activity, at any particular stage, affects circuit dynamics in various biological functions, including learning and memory processes. It also has great potentials for therapeutic applications.

\section{Author Contributions}

MTH designed experiments, analyzed the data and supervised the project. GKD prepared the viruses, performed the experiments and analyzed the data. MB generated unidirectional constructs and performed experiments. RA performed experiments and investigated the ITR effects. RS provided scientific input, discussion, and resources. GKD and MTH wrote the manuscript.

\section{Acknowledgments}

We thank Peter H. Seeburg for support. This work was supported by Max Planck Society and the Fritz Thyssen Stiftung (MTH) and the SFB636/A4 (RS).

Cambridge, S. B., Geissler, D., Calegari, F., Anastassiadis, K., Hasan, M. T., Stewart, A. F., et al. (2009). Doxycycline-dependent photoactivated gene expression in eukaryotic systems. Nat. Methods 6, 527-531. doi: 10.1038/ nmeth. 1340

Capecchi, M. R. (2005). Gene targeting in mice: functional analysis of the mammalian genome for the twenty-first century. Nat. Rev. Genet. 6, 507-512. doi: $10.1038 /$ nrg1619

Chen, C., and Okayama, H. (1987). High-efficiency transformation of mammalian cells by plasmid DNA. Mol. Cell. Biol. 7, 2745-2752.

Citri, A., and Malenka, R. C. (2008). Synaptic plasticity: multiple forms, functions, and mechanisms. Neuropsychopharmacology 33, 18-41. doi: 10.1038/sj.npp.1301559

Deisseroth, K. (2014). Circuit dynamics of adaptive and maladaptive behaviour. Nature 505, 309-317. doi: 10.1038/nature12982

Drouin, L. M., and Agbandje-McKenna, M. (2013). Adeno-associated virus structural biology as a tool in vector development. Future Virol. 8, 1183-1199. doi: $10.2217 /$ fvl.13.112 
Eschbach, J., and Danzer, K. M. (2014). Alpha-Synuclein in Parkinson's disease: pathogenic function and translation into animal models. Neurodegener. Dis. 14, 1-17. doi: 10.1159/000354615

Gossen, M., and Bujard, H. (1992). Tight control of gene expression in mammalian cells by tetracycline-responsive promoters. Proc. Natl. Acad. Sci. U.S.A. 89, 5547-5551. doi: 10.1073/pnas.89.12.5547

Hasan, M. T., Friedrich, R. W., Euler, T., Larkum, M. E., Giese, G., Both, M., et al. (2004). Functional fluorescent $\mathrm{Ca}_{2}{ }^{+}$indicator proteins in transgenic mice under TET control. PLoS Biol. 2:e163. doi: 10.1371/journal.pbio.0020163

Hasan, M. T., Hernández-González, S., Dogbevia, G., Treviño, M., Bertocchi, I., Gruart, A., et al. (2013). Role of motor cortex NMDA receptors in learningdependent synaptic plasticity of behaving mice. Nat. Commun. 4:2258. doi: $10.1038 /$ ncomms 3258

Hasan, M. T., Schonig, K., Berger, S., Graewe, W., and Bujard, H. (2001). Longterm, noninvasive imaging of regulated gene expression in living mice. Genesis 29, 116-122. doi: 10.1002/gene.1014

Hsiao, C. D., Hsieh, F. J., and Tsai, H. J. (2001). Enhanced expression and stable transmission of transgenes flanked by inverted terminal repeats from adeno-associated virus in zebrafish. Dev. Dyn. 220, 323-336. doi: 10.1002/dvd y.1113

Huganir, R. L., and Nicoll, R. A. (2013). AMPARs and synaptic plasticity: the last 25 years. Neuron 80, 704-717. doi: 10.1016/j.neuron.2013.10.025

Kandel, E. R., Dudai, Y., and Mayford, M. R. (2014). The molecular and systems biology of memory. Cell 157, 163-186. doi: 10.1016/j.cell.2014.03.001

McGee Sanftner, L. H., Rendahl, K. G., Quiroz, D., Coyne, M., Ladner, M., Manning, W. C., et al. (2001). Recombinant AAV-mediated delivery of a tet-inducible reporter gene to the rat retina. Mol. Ther. 3, 688-696. doi: 10.1006/mthe.2001.0308

Murphy, S. R., Chang, C. C., Dogbevia, G., Bryleva, E. Y., Bowen, Z., Hasan, M. T., et al. (2013). Acatl knockdown gene therapy decreases amyloid-beta in a mouse model of Alzheimer's disease. Mol. Ther. 21, 1497-1506. doi: 10.1038/mt.2013.118

Ramachandran, P. S., Keiser, M. S., and Davidson, B. L. (2013). Recent advances in RNA interference therapeutics for CNS diseases. Neurotherapeutics 10 , 473-485. doi: 10.1007/s13311-013-0183-8

Schnepp, B. C., Jensen, R. L., Chen, C. L., Johnson, P. R., and Clark, K. R. (2005). Characterization of adeno-associated virus genomes isolated from human tissues. J. Virol. 79, 14793-14803. doi: 10.1128/JVI.79.23.14793-14803.2005

Shaner, N. C., Steinbach, P. A., and Tsien, R. Y. (2005). A guide to choosing fluorescent proteins. Nat. Methods 2, 905-909. doi: 10.1038/nmeth819
Shimshek, D. R., Kim, J., Hübner, M. R., Spergel, D. J., Buchholz, F., Casanova, E., et al. (2002). Codon-improved Cre recombinase (iCre) expression in the mouse. Genesis 32, 19-26. doi: 10.1002/gene.10023

Soriano, P. (1999). Generalized lacZ expression with the ROSA26 Cre reporter strain. Nat. Genet. 21, 70-71. doi: 10.1038/5007

Sprengel, R., and Hasan, M. T. (2007). Tetracycline-controlled genetic switches. Handb. Exp. Pharmacol. 49-72. doi: 10.1007/978-3-540-35109-2_3

Stanford, W. L., Cohn, J. B., and Cordes, S. P. (2001). Gene-trap mutagenesis: past, present and beyond. Nat. Rev. Genet. 2, 756-768. doi: 10.1038/35093548

Tonegawa, S., Nakazawa, K., and Wilson, M. A. (2003). Genetic neuroscience of mammalian learning and memory. Philos. Trans. R. Soc. Lond. B Biol. Sci. 358, 787-795. doi: 10.1098/rstb.2002.1243

Urlinger, S., Baron, U., Thellmann, M., Hasan, M. T., Bujard, H., and Hillen, W. (2000). Exploring the sequence space for tetracycline-dependent transcriptional activators: novel mutations yield expanded range and sensitivity. Proc. Natl. Acad. Sci. U.S.A. 97, 7963-7968. doi: 10.1073/pnas.130192197

Wallace, D. J., Meyer zum Alten Borgloh, S., Astori, S., Yang, Y., Bausen, M., Kügler, S., et al. (2008). Single-spike detection in vitro and in vivo with a genetic $\mathrm{Ca}^{+}{ }^{+}$sensor. Nat. Methods 5, 797-804. doi: 10.1038/nmeth.1242

Wang, J., Faust, S. M., and Rabinowitz, J. E. (2011). The next step in gene delivery: molecular engineering of adeno-associated virus serotypes. J. Mol. Cell. Cardiol. 50, 793-802. doi: 10.1016/j.yjmcc.2010.10.017

Yogev, S., and Shen, K. (2014). Cellular and molecular mechanisms of synaptic specificity. Annu. Rev. Cell. Dev. Biol. 30, 417-437. doi: 10.1146/annurevcellbio-100913-012953

Zhu, P., Aller, M. I., Baron, U., Cambridge, S., Bausen, M., Herb, J., et al. (2007). Silencing and un-silencing of tetracycline-controlled genes in neurons. PLoS ONE 2:e533. doi: 10.1371/journal.pone.0000533

Conflict of Interest Statement: The authors declare that the research was conducted in the absence of any commercial or financial relationships that could be construed as a potential conflict of interest.

Copyright (c) 2015 Dogbevia, Marticorena-Alvarez, Bausen, Sprengel and Hasan. This is an open-access article distributed under the terms of the Creative Commons Attribution License (CC BY). The use, distribution or reproduction in other forums is permitted, provided the original author(s) or licensor are credited and that the original publication in this journal is cited, in accordance with accepted academic practice. No use, distribution or reproduction is permitted which does not comply with these terms. 